\title{
Essai de traitement de la Péripneumonie contagieuse du Bœuf par la Bronchocilline
}

\author{
par Hadj-Amadou CAMARA
}

\section{I. - CONSIDÉRATIONS PRÉLIMINAIRES}

La péripneumonie contagieuse du boeuf continue de poser, aux Vétérinaires d'Outre-Mer, des problèmes de prophylaxie médicale et sanitaire difficiles à résoudre. Curasson (1) ne déclarait-il pas, il y a déjà quatorze ans, que cette affection «est décevante au possible, aussi bien pour ceux qui la combattent que pour ceux qui l'étudient »? Aujourd'hui, la situation ne semble pas améliorée de façon sensible, malgré les recherches entreprises et malgré les acquisitions de la thérapeutique pendant ces dernières années. Tout récemment encore, Mornet (2) dans un important travail, a fait le point des divers traitements mis en œuvre contre la maladie et il est parvenu aux conclusions suivantes :

a) le novarsénobenzol demeure, à ce jour, la meilleure arme;

b) il y a lieu de continuer les essais avec les sulfamides et les antibiotiques.

A propos d'antibiotiques, nous avons connu la Bronchocilline par la thèse que Montlaur (3) a consacrée à l'étude de cette pénicilline, employee dans quelques maladies à localisations respiratoires. L'analyse de ce travail, parue dans la Revue vétérinaire de Toulouse, mettait l'accent sur un caractère pharmacodynamique particulier du produit : sa concentration élective dans le poumon.

Notant cette intéressante propriété, nous nous sommes demandé si l'arsenal thérapeutique ne venait pas d'être doté d'une médication de valeur, susceptible de rénover le traitement médical de la péripneumonie contagieuse du bœeuf. Celle-ci, cn effet, avait été jusqu'ici combattue, comme n'importe quelle autre grande infection, par l'emploi des antiseptiques généraux. D'ailleurs rien n'autcrisait à mener le traitement autrement, puisque la péripneumonie est une maladie microbienne, virulente et contagieuse.

Cette définition, fondée sur les données générales de la bactériologie, a permis de classer la péripneumonie parmi les grandes enzooties, mais a masqué, du même coup, un aspect particulier et typique de la maladie qui demeure avant tout et spécifiquement une affection du poumon. Ce caractere de stricte localisation - qui entraine une symptomatologie en bien des points semblable à celle des aulres pleuro-pneurnonies - devrait, à notre sens, dominer le débat et guider le traitement médical. Vu sous cet angle, le probleme revient à atteindre l'agent pathogène logé dans l'intimité du poumon.

A priori, on peut donc assez raisonnablement envisager qu'un médicament bactéricide, qui s'accumule dans le poumon ou qui s'élimine par lui, pourra manifester un certain pouvoir curatif.

Telle est l'hypothèse que, devant les faits cliniques de la péripneumonie, nous avons été amené à formuler et que nous avons voulu soumettre à la vérification expérimentale. Il semble même possible d'affirmer, d'un point de vue plus général, que tout médicament actif in situ contre l'infection pulmonaire (abcès, tuberculose, par exemple), devrait l'être également contre la péripneumonie.

La Bronchocilline est un antibiotique qui répond aux deux conditions requises, formulées ci-dessus; il ne lui manque que la sanction de l'expérience. Avant de parler de nos essais - malheureusement trop peu nombreux à notre gré - et que, pour cette raison, nous avons voulu aussi complets que possible, nous pensons utile de rappeler les caractères et les propriétés de la Bronchocilline, ce que nous ferons par de larges extraits de la thèse de Montlaur.

\section{II. - LA BRONCHOCILLINE}

«Le Professeur Jensen de Copenhague et ses collaborateurs, écrit Montlaur, réalisent la synthèse de plusieurs esters de la benzyl-péniciline. L'un de ces esters injecté chez l'animal, ne se retrouve dans le sérum sanguin qu'en faible quantité et cependant n'est pas éliminé par les urines. Une etude systématique de cette curieuse propriété 
permet de mettre en évidence une concentration élective de l'antibiotique dans le tissu pulmonaire.

La Bronchocilline est l'iodhydrate de l'ester diéthyl-arninu-éthylique de la benzyl-pénicilline.

La pénicilline est un diacide répondant à la formule suivante:

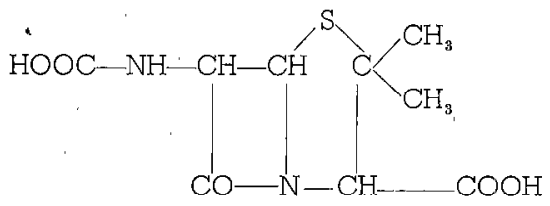

Dans cette formule" Polonowski reconnaît le squelette de deux acides aminés, la cystéine et la valine :<smiles>NC(CS)C(=O)O</smiles>

cystéine<smiles>CC(C)C(N)C(=O)O</smiles>

valine
La pénicilline $G$ ou benzyl-pénicilline présente un radical benzyl $\mathrm{C}_{6} \mathrm{H}_{5}-\mathrm{CH}_{2}$ greffé sur l'une des fonctions acides.

L'autre fonction acide étant estérifiée par le diéthyl-amino ćthanol :<smiles>[C]CCCN(CC)CC</smiles>

on obtient l'ester diéthyl-amino-éthylique de la benzyl-pénicilline :<smiles>CCN(CC)CCOC(=O)C1N2OC(=O)C(SC1(C)C)C2(C)CC[AsH3+]</smiles>

Cet ester présente une certaine parenté chimique avec la pénicilline-procaine puisque la procaïne possède le noyau diéthyl-amino-éthylique.

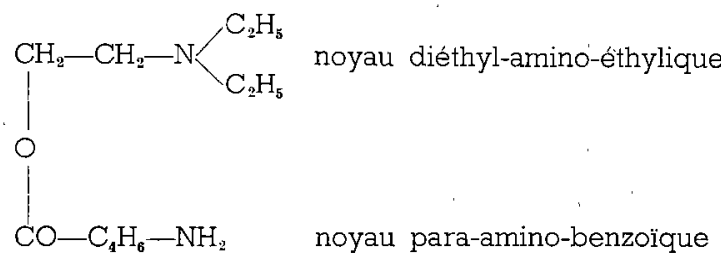

Propriété biochimique remarquable quil explique sa concentration élective, la Bronchocilline est estérifiée presque uniquement dans le poumon en libérant la benzyl-pénicilline.

\section{Conclusions de l'étude pharmacologique:}

$1^{\circ} \mathrm{La}$ Bronchocilline se concentre électivement dans le tissu pulmonaire:

$2^{\circ}$ Elle s'élimine par les expectorations :

$3^{\circ}$ Elle possède un effet retard moyen ( 10 heures);

$4^{\circ} \mathrm{La}$ localisation pulmonaire de l'antibiotique se fait aux dépens des autres organes et du sérum sanguin en particulier. "

Or, dans la peripneumonie du bœuf; le virus jouit d'un tropisme exclusif vis-à-vis du poumon, au détriment des autres organes. Il èst vrai que, dans certains cas, le sang se montre virulent,' mais ce caractère demeure inconstant. Ces considérations permettent de préjuger de l'activité de la Bronchocilline; elles justifieront l'efficacité du novarsénobenzol, par l'action eupnéique de l'ion arsenical ; elles expliqueront enfin l'échec de la Spécilline qui se concentre presque exclusivement dans le sang.

\section{III. - EXPÉRIENCES $\left({ }^{*}\right)$}

Notre intention première était de comparer l'action de la'Bronchocilline, de l'auréomycine et de la terramycine, suivant les conseils de M. le Professeur Verge. Nous n'avons pu malheureusement nous procurer de la terramycine et la faible quantıté dont nous disposions des autres antibiotiques a réduit l'ampleur que nous voulions donner à nos essais.

Afin. d'employer nos produits au maximum, nous avons choisi, avec l'accord des propriétaires, 5 animaux parmi les plus gravement atteints, dans le foyer de Nounkounkan (canton de Koulounkalan, cercle de Siguiri) et nous avons adopté le protocole suivant :

No 1, Vache : deux poumons atteints; auréomycine :

No 2 , Vache : deux poumons atteints; bronchocilline:

No 3, Génisse : un poumon atteint; bronchocilline;

(*) Nous sommes heureux de remercier ici le Vétérinaire afriçain principal Demba Julien, en service à Siguiri, qui a été pour nous un précieux collaborateur dans les relevés et observations cliniques.

Nos remerciements vont encore aux Laboratoires VET-ORGA et SPECIA (Agence de Dakar), qui ont bien voulu mettre à notre disposition les produits employés dans nos expériences.

Nous remercions enfin nos Maîtres MM. les Professeurs Verge et Darraspen, qui nous ont guidé de leurs conseils. 
No 4, Bœuf de labour : deux poumons atteints association bronchocilline-spécilline ;

No 5, Vache : un poumon atteint; assoziation bronchocilline-spécilline.

Le traitement était effectué en trois jours, du 6 au 8 août 1954, avec injection unique pendant la première journée et deux injections par jour, matin et soir, pendant les deux jours suivants.

Des examens cliniques ont eu lieu les 6,9 et 21 août, puis un mois après le début du traitement, le 7 septembre. Enfin un dernier contrôle pour bilan a pu être effectué le 24 février 1956, soit un an et demi après les interventions.

Voici le détail de nos observations. Nous nous excusons de n'avoir pas joint les graphiques de températures, les relevés ayant été poursuivis pendant plus d'un mois.

\section{Observation no 1}

Vache, 8 ans.

6 août 1954:

Examen clinique : $\mathrm{T}=.41^{\circ} 2$; dyspnée, toux.

Auscultation.: signes indiquant une atteinte des deux poumons.

Percussion : matité et sensibilité des deux côtés, plus accusées à droite.

6 aout : injection intraveineuse de $400 \mathrm{mg}$ d'auréomycine. La.dyspnée est telle que l'injection doit être faite sur l'animal debout.

Températures : matin, $41^{\circ} 2$; soir, $41^{\circ} 2$.

7 août : injection de $400 \mathrm{mg}$ d'aurćomycine lo matin; répétition de la même dose le soir.

Températures : matin, $40^{\circ} \mathrm{l}$; soir, $40^{\circ} 2$.

8 août : même traitement.

Températures : matin, 4003 ; soir, $40^{\circ} 7$.

9 août :

Examen clinique: poils hérissés, pouls veineux, dyspnée avec plaintes.

Auscultation : silence du poumon droit, souffle tubaire à gauche.

Percussion : sensibilité costale des deux côtés, matité plus accusée à droite, en zone inférieure. Pronostic assombri.

Températures : matin, $39^{\circ}$; soir, $41^{\circ} 2$.

Le 12 août, on notait encore les températures suivantes : $3903-41^{\circ}$; le $15: 3803-40 \circ 9$; le 17 : $38^{\circ}-4105$; le 19 : $39^{\circ}-4102$; le 21 : $39^{\circ}-39^{\circ}$.

21 août :

Examen clinique : «Poil piqué ».

Auscultation : à droite, souffle tubaire à la base du poumon, murmure vésiculaire voilé dans le lobe antérieur ; à gauche, bruit de frottement pleural.

Percussion : à droite, matité, plus accusée au sommet; à gauche, matité faible.

La sensıbilité costale est plus prononcée à droite.

La température rectale notée chaque matin, du
2. août aı 7 septembre, était peu différente de la normale, mais elle s'élevait chaque soir, oscillant entre $39^{\circ} 6$ et $40^{\circ} 9$. Ceci indiquait que la maladie n'était pas encore jugulée, puisque les rémissions matinales étaient toujours suivies d'exacerbations vespérales. Cette aggravation doit, du reste; être mise sur le compte de l'inclémence de la saison, car les mois d'août et septembre marquent l'acmé des précipitations d'eau.

7 septembre:

Examen clinique: animal maigre, hanches saillantes, poils hérissés, toux rauque, appétit irrégulier, inrumination.

Épreuve de la course : respiration et circulation accélérées, essoufflement prononcé.

Auscultation : à droite, souffle tubaire voilé ; à gauche, exagération du murmure vésiculaire.

Percussion : matité des deux côtés, sensibilité costale plus accentuée à droite.

Il apparait donc, un mois après le traitement, que la dose a été manifestement insuffisante pour arrêter la progression des lésions qui semblent simplement retardées.

L'animal qui, pourtant, a guéri par la suite, a été livré en janvier 1956 à la boucherie; les poumons ne présentaient pas de lésions macroscopiques.

\section{Observation $n^{\circ} 2$}

Vache, 8 ans.

6 aout 1951 :

Examen clinique: toux, pouls veineux, $\mathrm{T}=41^{\circ} \mathrm{C}$.

Auscultation : souffle tubaire franc à droite, souffle voilé à gauche.

Percussion : matité et sensibilité des deux côtés.

Injection intramusculaire d'un million d'unités de Bronchocilline.

Températures : matin, $41^{\circ}$; soir, 4105.

7 août : injection matin et soir d'un million d'unités de Bronchocilline.

Températures : matin, 3907 ; soir, $41^{\circ} 3$.

8 aout : même traitemenl.

Températures : matin, $39^{\circ}$; soir, $41^{\circ} 3$.

9 août :

Examen clinique: Toux sèche, courte, appétit et rumination revenus ; poil luisant.

Auscultation et percussion : résultats sans changement.

Du 10 au 15 août, les températures matinales sont passées de $40^{\circ}$ à $38,3^{\circ} \mathrm{C}$; mais les tempćratures vespérales ont été de $40^{\circ}$ à $41^{\circ} 2$.

Ce n'est qu'à partir du 18, soit 13 jours après le début du traitement que la température redevient normale. On observe par, la suite, un léger retour offensif et l'hyperthermie vespérale ne disparaîtra que le 4 septembre (29e jour). 
21 août :

Examen clinique : Poil piqué.

Auscultation : à droite, souffle tubaire moins inlerise ; à gauche, bruit de frottement pleural.

Percussion : matité et sensibilité atténuées des deux côtés.

7 septembre:

Examen clinique: Poils hérissés, toux peu fréquente et intermittente, appétit et rumination rétablis.

Auscultation : à droite, léger souffle tubaire; à gauche, bruit de crépitement.

Percussion : résonance presque normale des deux côtés, mais sensibilité costale non encore complètement disparue.

Épreuve de la course : léger essoufflement, légère accélération circulatoire.

$\mathrm{Ce}$ cas, qui était très grave (péripneumonie, avec pleurésie exsudative) est très nettement amélioré bien qu'il persiste encore des séquelies de la maladie.

29 février 1956 :

Examen clinique : vache en parfait état sanitaire et d'entretien, a vêlé en décembre 1955.

\section{Observation $n^{\circ} 3$}

Génisse. 3 ans.

6 août 1954 :

\section{Examen clinique :}

Auscultation : murmure vésiculaire exagéré à droite :

Percussion : matité et sensibilité du côté droit ; Poumon gauche normal.

Injection d'un million d'unités de Bronchocilline en intra-musculaire.

Températures : matin, $41^{\circ}$; soir, $41,4^{\circ} \mathrm{C}$.

7 août : injection d'un million d'unités de Bronchocilline matin et soir.

Températures : matin, 3904 ; soir, 4102.

8 août : même traitement.

Températures : matin, 3805 ; soir, $40^{\circ} 7$.

9 août:

Examen clinique: poil luisant, appétit et rumination revenus.

Auscultation : souffle tubaire à droite.

Percussion : sensibilité et matité du même côté.

Poumon gauche : R.A.S.

Températures : matin, $38^{\circ}$; soir, $41^{\circ} 3$.

L'influence du traitement est immédiate et se traduit par une chute progressive des temperatures matinales et vespérales, abstraction faite du relèvement du soir du $3^{\text {e jour. }}$

- Du 10 au 15 août, les températures matinales ont oscillé entre $39^{\circ} 7$ et $38^{\circ} 6$, celles du soir entre $40^{\circ} 8$ et $41^{03}$ avec une montée à 4109 le 12. Du 16 au 21 août, la température de l'animal presque normale le matin. montait le soir à $39^{\circ} 6^{\circ}-39^{\circ} g$ et $40^{\circ} 1$.
Le $16^{e}$ jour après le traitement seulement, la température vespérale qui a baissé très irrégulièrement, avec des retours offensifs, est "redevenue normale.

21 août :

Examen clinique: poil luisant, toux courte et sèche, appétit et rumination bons.

Auscultation : souffle tubaire atténué, 'à droite.

Percussion : légère sensibilité costale à droite.

Poumon gauche : R.A.S.

Du 22 au 29 août, on a pu noter encore des températures atteignant $40,7^{\circ} \mathrm{C}$ le soir mais la tendance à la baisse a été assez régulière et, le 7 septembre, on pouvait relever une température rectale de $38,8^{\circ} \mathrm{C}$, le soir.

7 septembre :

Examen clinique: poil luisant, bon état général, appétit et rumination excellents, toux disparue.

Auscultation et percussion : R.A.S.

Épreuve de la course : pas d'essouflement, pas d'accélération circulatoire.

ANIMAL GUÉRI.

Examen clinique du 24 février 1956 : en parfait état de santé ; actuellement pleine, est prête à vêler.

\section{Observation $n^{\circ} 4$}

Boeuf de labour 4 ans.

6 août 1954 :

\section{Examen clinique :}

Auscultation : frottement pleural à droite ; soufîle tubaire à gauche.

Percussion : matité et sensibilité des deux côtés.

Sur cet animal, on essaye la Bronchocilline et la Spécilline associées, en injections alternées; on espère, de la sorte, neutraliser, le cas échéant, la virusémie.

Le premier jour du traitement, l'animal reçoit par voie musculaire un million d'unités de Bronchocilline.

Températures : matin, $40^{\circ} 7$; soir, $41^{\circ} 8$.

7 août : le matin un million d'unités de. Bronchocilline ; le soir, même dose de Spécilline, même voie d'administration.

Températures : matin, 3803 ; soir, $41^{\circ} 2$.

8 août : même traitement que la veille.

Températures : matin, $38^{\circ}$; soir, 4004.

9 aout :

Examen clinique: poils hérissés, appétit et rumination irréguliers, mauvais état général.

Auscultation : frottement pleural persistant à droite; souffle tubaire à gauche.

Percussion : matité franche du côté droit ; foyers de résonance (suppléances) à gauche; sensibilité des deux côtés, plus accusée à droite.

Températures : matin, $38^{\circ} 2$; soir, $41^{\circ} 1$. 
Les jours suivants, l'animal a présenté les températures suivantes :

10 août : matin, 3909 ; soir, 3905.

11 août : matin, 3605 ; soir, 3908.

12 août : matin, 3403 ; soir, mort.

A l'autopsie, on constate l'hépatisation massive du poumon droit et des illots de pneumonie dans le poumon gauche.

Dans ce cas, qui rappelle en gravité l'observation no 2 (pneumonie, avec pleurésie exsudative et atteinte des deux poumons), l'association Bronchocilline-Spécilline s'est révélée beaucoup moins active que la Bronchocilline seule. Cela n'a rien d'étonnant, en raison du manque d'activité de la Specilline : Mornet et coll. (2) ont injecté sans résultat de la pénicilline $\mathrm{G}$ en solvant-retard, à la dose d'un million à trois millions d'unités dans la journée.

\section{Observation no 5}

Vache, 4 ans.

6 août 1954 :

Examen clinique : toux avec dyspnée.

Auscultation : souffle tubaire à droite.

Percussion : matité costale du même côté.

Poumon gauche : aucun signe.

Cet animal subit le même traitement, aux mêmes doses et selon les mêmes modalités que dans l'observation $n^{\circ} 4$ : un million d'unites de Bronchocilline ou de Spécilline en injections alternées.

Relevé des températures:

$\begin{array}{ccc}\text { Dates } & \text { Matin } & \text { Soir } \\ 6 \text { août : } & \overline{41^{\circ} 1} & 41^{\circ} 5 \\ 7 \text { août : } & 39 \circ 7 & 41^{\circ} 1 \\ 8 \text { août : } & 39 \circ 2 & 41^{\circ} \\ 9 \text { août : } & 38^{\circ} 7 & 41^{\circ} 1\end{array}$

La température matinale, qui était élevée au début, ne tarde pas à marquer une chute notable, mais la température vespérale se maintient toujours élevée

9 août :

Examen clinique: toux sèche et courte, appétit et rumination revenus.

Auscultation : souffle tubaire à droite.

Percussion : matité et sensibilité à droite.

Poumon gauche : R.A.S.

Les ascensions vespérales de la température continuent à se manifester jusqu'au 15 aout $\left(40^{\circ}\right)$.

Du 16 au 21, la baisse de la température vespérale, quoique lente et irrégulière, est néanmoins très nette. La température normale est atteinte le 13e jour: mais elle remonte des le lendemain et il faut mettre cette aggravation sur le comple des pluies.

21 août :

Examen clinique : poil luisant, meilleur état général, toux sèche et persistante.
Auscultation : léger souffle tubaire à droite.

Percussion : légère matité et sensibilité du même côté.

Par la suite, on ne notera qu'une fois (26 août) une nouvelle montée de la température vespérale à $40,3^{\circ} \mathrm{C}$. Aucun écart thermique significatif n'a été observé du 27 août au 7 septembre.

7 septembre :

Examen clinique: bon embonpoint, poil luisant, bon état général, appétit et rumination excellents.

Auscultation et percussion : aucun signe.

Épreuve de la course : pas d'essoufflement, pas d'accélération circulatoire.

L'animal paraît guéri.

Examen clinique du 24 février 1956 : animal en excellent état sanitaire, a repris de l'embonpoint, a vêlé en décembre 1955.

\section{IV. - CONCLUSIONS}

Les essais de traitement ont été entrepris intentionnellement dans les conditions les plus défavorables de la pratique de brousse. Après triage des malades à soumettre à l'expérimentation, on n'a retenu de ceux-ci que les sujets présentant les signes les plus graves accompagnés d'hyperthormic et chez lesquels, vraisemblablement, le novarsénobenzol aurait échoué.

A cela, il y avait plusieurs raisons : en premier lieu, le désir d'éprouver des produits qui, pour être intéressants, devraient se révéler plus actifs que le 914, ainsi que les limites très strictes que nous imposait la faible quantité de médicaments à notre disposition,

Ensuite, nous avons expérimenté en pleine saison des pluies, en août et septembre alors que la pluviométrie est maxima. Nous avons voulu faire jouer contre nos expériences le facteur d'aggravation que constitue l'action de continuelles précipitations d'eau sur des malades pulmonaires soumis à toutes les intempéries. Si cette action constante s'est souvent vérifiée sur les températures vespérales, la saison a eu, par contre, une action favorable par l'abondance de la nourriture et de l'abreuvement.

Enfin nous avons dû employer, et toujours pour des raisons de pénurie de médicaments, des doses minima actives qui ont donné les résultats déjà enregistrés et qui ont été injectées dans les conditions ordinaires de la pratique tropicale, en deux jours et demi.

Le dernier examen clinique, en date du 26 février 1956, intervenu plus d'un an et demi après les essais, a pu légèrement modifier nos conclusions, déjà écrites, et qui en sont devennes 
plus formelles, etant mieux assises, mais le sujet de cette note, qui ne mentionnait pas l'auréomycine, a été maintenu tel quel.

C'est que, dans l'ignorance du sort des sujets d'expérience, nous avions conclu, après une observation d'un mois, que l'auréomycine, à' la dos $\theta$ employée, prolongeait simplement la survie du malade, car nous étions persuadé de l'issue fatale dans un cas si avancé qu'il a tallu faire les injections sur l'animal debout, le décubitus pouvant entraîner I'asphyxie. Cet animal a quéri, mais plus lentement que les autres, et on se demande quelle part a pu d'ailleurs prendre, dans cette guérison, la foi avec laquelle le propriétaire a entouré le malade de soins particulièrement attentifs.

En ce qui concerne la Bronchocilline, les doses employées dans nos expériences sont infimes par rapport à la marge de tolérance et aussi par rapport aux doses injectées dans certains cas : chez l'homme, par exemple, il'n'a pas fallu moins de deux millions d'unités par jour, injectées pendant vingt jours consécutifs, pour venir à bout d'un abcès pulmonaire, alors que la péripneumonie du bouf est vaincue par la même dose utilisée pendant deux jours et demi seulement, soit, en fin de compte, une dose totale huit fois moindre chez l'animal que chez l'homme.

Les résultats obtenus ont été la guérison de trois malades sur quatre traités à la Bronchocilline. Dans deux cas, la maladie n'atteignait qu'un seul poumon et, à ce stade, l'association BronchocillineSpécilline s'est révélée avantageuse, car elle permet de réduire de moitié la dose de Bronchocilline. Mais lorsque la maladie s'est étendue aux deux poumons, la Bronchocilline employée seule, améliore et finit par guérir le sujet, tandis que l'association Bronchocilline-Spécilline est impuissante à enrayer le processus, ce qui n'a rien de surprenant, vu l'inactivité de la Spécilline.

L'expérimentation n'est pas toujours facile, en matip̀re de péripneumonie, en raison des nombreuses incertitudes qui planent sur la pathogénie de l'afféction et sur la virulence du microorganisme. La comparaison des résultats obtenus avec différents produits n'est possible que si les produits ont été injectés à des malades parvenus au mème stade clinique; c'est souligner du même coup l'importance que revêt, dans ce cas, l'examen des signes stéthoscopiques et plessimétriques présentés par les sujets d'expérience.

Parmi les antibiotiques, la Bronchocilline semble devoir autoriser de grands espoirs, mais des espoirs tempérés de scepticisme. C'est que la péripneumonie est une maladie décevante pour ceux qui, n'ayant pas la possibilité de s'en débarrasser par le stamping out, sont obligés de ła combattre médi- calement. Or, dảns cette lutte, on a été déjà le témoin de médications dont les essais ont débuté dans l'enthousiasme et ont abouti à la déception. Nous avons particulièrement en vue le cas du lipo-vaccin. Il y aura bientôt vingt ans que, répondant à un ques-stionnaire de l'Inspection générale sur' lcs moyens de lutte à employer contre la péripneumonie, nous avons préconisé l'usage d'un lipo-vaccin. Comme nous ne disposions pas de moyens expérimentaux, étant praticien de la brousse, nous nous sommes contenté d'étudier théoriquement le lipo-vaccin (4). Nous ignorions; du reste, les recherches, sur le même sujèt entreprises par Dischamps à Bamako (cité par Curasson). Les expériences du lipovaccin, d'abord prometteuses, durent être abandonnées par la suite.

Ce rappel nous remet en mémoire cette période que nous serions tenté de qualifier d' «héroïque » pour nous, car elle marque les premiers contacts, plutôt rudes, que nous avons prịs avec la péripneumonie. De cette époque est restée, du moins la notion d'allergie dans la péripneumonie dunt nous avons'eu le pressentiment, devant le témoignage des faits cliniques (5).

En résumé, et, en mettant de côté toute question de prix, il paraît assez logique, d'après les premiers résultats obtenus, d'avoir recours à la Bronchocilline pour traiter la péripneumonie et les premiers résultats expérimentaux y autorisent. Mais l'avenir, qui est le Grand Maître, dira quel crédit il faut accorder à cet antibiotique.

\section{Service de l'Élevage de la Guinée Française.} Circonscription d'Élevage de Haute-Guinée.

\section{BIBLIOGRAPHIE}

1. CURASSON (G.). - Traité de pathologie exotique. $2^{\mathrm{e}}$ édition, 1942, Vigot édit., Paris.

2. MORNET (P.): - Traitement de la péripneumonie bovine. Bull. des Épiz. en Afrique (1954), 2, 27. Voir aussi : Bull. Techn. d'inform. de l'Institut d'Élev. et de Méd. Vétér. des Pays Tropic. no 2, '1954; 39.

3. MONTLAUR (P.). - La Bronchocilline dans le traitement de quelques localisations respiratoires de la maladie de Carré et de la gourme. Thèse vétér.; Toulouse (1952). Analyse dans Rev. Méd. 'Vét, Toulouse (1953), 104; 299.

4. CAMARA, (A.). - Étude theorique d'un lipovirus dans le traitement de la péripneumonie. Protocole expérimental. Note inédite du 25 Novembre 1937.

5. CAMARA (A.). - Étude de l'allergie dans la péripneumonie. Note inédite du 17 Octobre 1938. 


\section{SUMMARY}

A test treatment of contagious bovine pleuropneumonia with «Bronchocilline »

In Senegal, during the rainy season, five cattle were treated with Bronchocilline. Doses of 1.000.000 units were injected intramuscularly morning and evening for two days.

The clinical examination of the same animals carried out several months later showed the valuable results of this treatment. In only one case did this treatment fail to give appreciable results.

Post mortem examinations carried out on animals given up for slaughter permitted verification of the disappearance of the macroscopic pulmonary lesions.

\section{RESUMEN}

\section{Ensayo de tratamiento de la perineumonía contagiosa del buey por la «Broncocilina »}

En el Senegal, durante la estación de las lluvias, cinco bovinos hlan sido tralados por la « broncocilina », con una dósis de 1.000 .000 de unidades en inyecciones intramusculares repetidas mañana y tarde durante dos dias.

El exámen clínico de estos mismos animales, efectuado varios meses más tarde muestra el valor de esta terapeutica. Sólamente ha fracasado la medicación en un animal. Los exámenes efectuados postmortem en animales entregados al matadero permiten advertir la desaparición de las lesiones macroscópicas pulmonares. 